\title{
A Resource Allocation Problem Based on GA-PSO Algorithm
}

\author{
Guo Zheyu \\ North China Electric Power University Baoding, Baoding 071000, China \\ 490931035@qq.com
}

\section{Keywords: SOM neural network, GA-PSO algorithm, dynamic subsidy}

\begin{abstract}
This paper proposes a scheme for resource allocation of taxi. Using SOM neural network divides the area into several areas, and calculating taxi transfer number for each area by using a method of dynamic subsidy and GA - PSO algorithm. Before and after comparing with the scheme of the taxi demand matching degree, and we find the matching degree has been greatly improved finally.
\end{abstract}

\section{Introduction}

Nowadays, with the improvement of living standards, more and more people tend to choose a taxi as one of the transportation mode. In the Internet era, the taxi software platform based on network technology development in many markets, and it is very convenient for taxi drivers and passengers between instant communications, which changes the drivers from carrying passenger aimlessly to pull into a targeted and carrying out. At the same time, these companies launched every kind of the taxi subsidy scheme. The introduction of subsidies scheme greatly affected the passenger taxi drivers' choice. This article will take Beijing as an example, discuss the taxi resource allocation problems, and give a taxi subsidy scheme.

Using a large data intelligent mobile travel platform "sky" ( $h$ ttp://v.kuaidadi.com/) to collect taxi amount and taxi distribution demand in Beijing in various locations at the same time, and delete the abnormal data, and each point of latitude and longitude values into Cartesian coordinates in order to measure the distance between points. Due to the statistical location data collected included too much, this paper uses SOM neural network statistical dimension, and Beijing will be divided into 40 regions.

Using those collected data, combined with the distance between the regional centers for solving the resulting, structural parameter transfer function matrix and the two kinds of indexes respectively corresponding standard 0-1 transformation. For the standardization of the matrix, taking each index maximum value, and getting the optimal subsidy programs, which are the subsidies to the amount of the maximum number of subsidy schemes.

We can analyze the gray weighted associations of the subsidy scheme, and the ideal optimal subsidy scheme by using the grey correlation analysis. According to the gray weighted association degree, we sort the different subsidy scheme, and if the rank is well, the amount of the subsidies is great. Considering the actual program development process, it is not possible for any situation are designed corresponding to the amount of subsidy. Therefore, according to the size of the grey weighted related degree, subsidy program is divided into 5 categories, the amount of subsidy respectively 17 Yuan, 13 Yuan, 10 Yuan, 8 Yuan, 6 Yuan, and gives the corresponding subsidy scheme. Thus, dynamic subsidy scheme on the basis of the different spatial and temporal changes of the taxi distribution and taxi demand quantity and the build is completed.

Using the passenger benefit analysis model and we can get the benefit transfer under dynamic subsidy scheme. Substituting the second issue of taxi transfer model, using algorithm GA-PSO optimization method, and with the help of MATLAB simulation, and we can get dynamic subsidy scheme under the influence of the taxi transfer.

Access to information, the vehicle's average speed is $15.9 \mathrm{~km} / \mathrm{h}$ and taxi driver in Beijing received 5000 Yuan monthly. Assuming a month has 30 days, average working 12 hours a day, so the Beijing taxi driver's income is 13.9 Yuan average per hour, which is also to say 1 hour 
equivalent to 13.9 Yuan. Beijing taxis are gas dual-purpose now, and the average fuel (gas) fee is 0.8 Yuan per kilometer. To sum up, we can obtain the benefit transfer expression as follow:

$$
c_{i j}=-\frac{l_{i j}}{15.9} \times 13.9-0.8 l_{i j}+b_{i j}
$$

In the formula, $c_{i j}$ says the taxi transfer efficiency by $i$ region metastasis to region $j ; l_{i j}$ is the distance between regions $i$ and $j ; b_{i j}$ says amount of taxi subsidies by region $i$ transferred to $j$.

Using the program before and after, each area's supply and demand indexes are as shown in figure 1 and figure 2. Comparing the index before with after, we can find that the demand has been greatly met.

In this formula, $l_{i j}$ is the distance between the center of region $i$ and the $j$, the Cartesian $\left(x_{i}, y_{i}\right)$ is the center of region $i$.

For any area, the number of taxi outflow area cannot over the sum of the number of the taxi driving into the area and originally the area containing the number of taxi. The specific expression is as follow:

$$
\sum_{i=1}^{40} z_{j i}-\sum_{i=1}^{40} z_{i j} \leq n_{j} \quad j=1,2, \ldots, 40
$$

Where $z_{i j}$ is the amount of taxis transfer from $i$ to $j ; n_{j}$ is the original number of taxis in the area.

Because taxi drivers' choice of go which place are determined by the transfer benefit, and the more benefit they can get, the more possibility they want to go there. If there is no benefit, the drivers are not willing to go to the place. The expression is as follow:

$$
\text { if } c_{i j} \leq 0, \quad z_{i j}=0
$$

Where $c_{i j}$ is the benefit drivers can get when transforming from area $i$ to $j$.

In view of transfer benefit is one of the biggest driving forces for the transfer of drivers, drivers are not willing to go to a place where the taxi number can meet the demand, that is also to say the transfer benefit is low. In other words, the amount of taxis will not be more than demand locally. We can get the expression as follow:

$$
\sum_{i=1}^{40} z_{j i}-\sum_{i=1}^{40} z_{i j}+n_{j} \leq m_{j} \quad j=1,2, \ldots, 40
$$

In this formula, $m_{j}$ is the demand of area $j$.

Assuming the number of taxis is a constant, it is also to say taxis only transform from one place to other place, and the total don't change, we can get another expression:

$$
\sum_{j=1}^{40}\left(\sum_{i=1}^{40} z_{i j}-\sum_{i=1}^{40} z_{j i}+n_{j}\right)=\sum_{j=1}^{40} n_{j}
$$

Taxis' transfer aim is to get biggest benefit, so the object is to get the maximal transfer benefit, and the objective function can be expressed as follow:

$$
\max \sum_{i=1}^{40} \sum_{j=1}^{40} c_{i j} \cdot z_{i j}
$$

In summary, we can get taxis transfer model: 


$$
\begin{aligned}
& \max \sum_{i=1}^{40} \sum_{j=1}^{40} c_{i j} \cdot z_{i j} \\
& \left\{\begin{array}{l}
\sum_{i=1}^{40} z_{j i}-\sum_{i=1}^{40} z_{i j} \leq n_{j} \quad j=1,2, \ldots, 40 \\
\text { if } c_{i j} \leq 0, \quad z_{i j}=0 \\
\sum_{i=1}^{40} z_{j i}-\sum_{i=1}^{40} z_{i j}+n_{j} \leq m_{j} \quad j=1,2, \ldots, 40 \\
\sum_{j=1}^{40}\left(\sum_{i=1}^{40} z_{i j}-\sum_{i=1}^{40} z_{j i}+n_{j}\right)=\sum_{j=1}^{40} n_{j}
\end{array}\right.
\end{aligned}
$$

In this formula, $z_{i j}$ is taxi number transferred from area $i$ to $j, n_{j}$ is the original taxi number in area $j, c_{i j}$ is benefit drivers can get when transferring from $i$ to $j$, and $m_{j}$ is the demand of taxis in area $j$.

Through the above model, we can solve the number of taxi transfer in each area. For judging if the taxi resource allocation is improved, we defined the supply and demand matching index:

$$
r_{i}=\frac{m_{i}}{n_{i}} \quad i=1,2, \ldots, 40
$$

Using the program before and after, each area's supply and demand indexes are as shown in fig. 1 and fig.2. Comparing the index before with after, we can find that the demand has been greatly met.

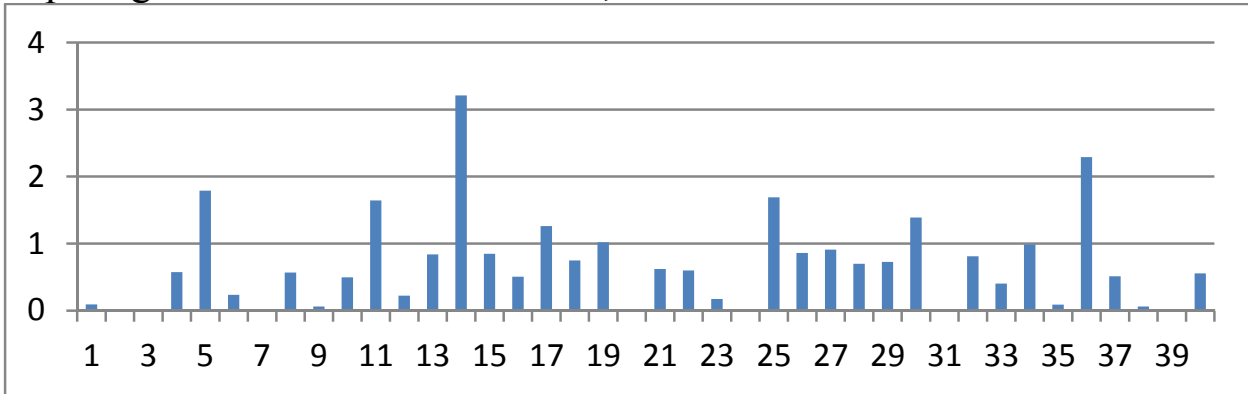

Fig.1 Supply and demand indexes without plan

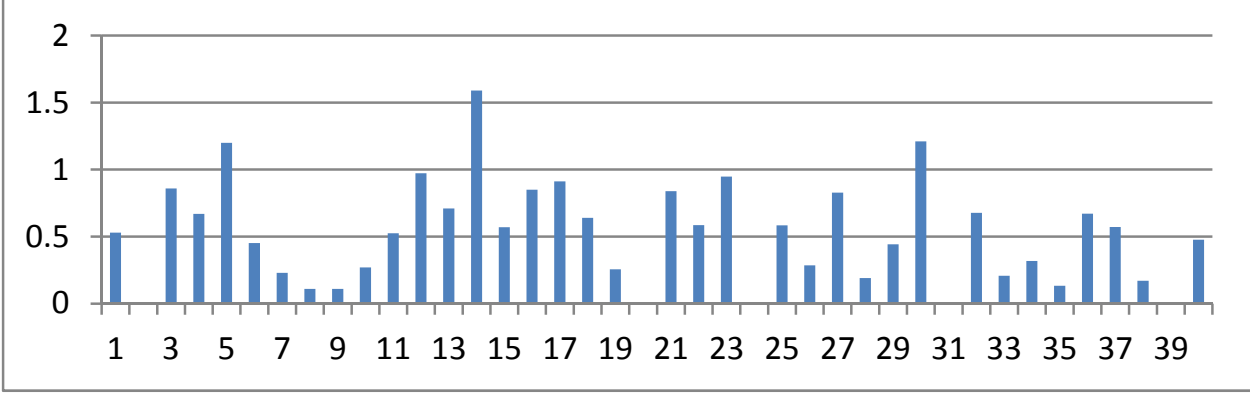

Fig.2 Supply and demand indexes with plan

\section{Conclusions}

While ensuring the taxi benefits, the method effectively improves the taxi resource allocation problem, and taking Beijing as example, we test the model and find it is rational. This model not only can be used in Beijing, but also can be used in other cities or regions.

\section{References}

[1] Yang Jianrong, The construction of our country city taxi industry characteristics analysis and demand forecasting model -- a case study of Nanchang City, Chinese Master's Theses Full-text Database, 2011, No.S1. 
[2] Zhang Yan, The taxi demand model for Chinese provinces, Public Business, 2008, 22: 45-47.

[3] Che Lan, The city taxi development and demand forecast, Shan Xi science and technology, 2006(06). 